\title{
Measurement of the W-boson mass at the ATLAS experiment
}

\section{Oleh Kivernyk}

LAPP, Univ. Grenoble Alpes, Univ. Savoie Mont Blanc, CNRS/IN2P3, Annecy

E-mail: oleh.kivernyk@cern.ch

We present the results of $W$-boson mass measurements with the ATLAS detector at the LHC based on the 2011 data set recorded at a centre-of-mass energy of $\sqrt{s}=7 \mathrm{TeV}$, and corresponding to $4.6 \mathrm{fb}^{-1}$ of integrated luminosity. The selected data sample consists of $7.8 \times 10^{6} \mathrm{~W} \rightarrow \mu \mathrm{v}$ candidates and $5.9 \times 10^{6} \mathrm{~W} \rightarrow e v$ candidates. Measurements are performed through template fits to transverse momentum distributions of charged leptons as well as to transverse mass distributions of the $W$ boson in electron and muon decay modes in various kinematic categories. All the individual measurements are found to be consistent and their combination leads to a value of

$$
\begin{aligned}
m_{W} & =80370 \pm 7 \mathrm{MeV} \text { (stat. }) \pm 11 \mathrm{MeV} \text { (exp.sys. }) \pm 14 \mathrm{MeV} \text { (mod.sys.) } \\
& =80370 \pm 19 \mathrm{MeV} .
\end{aligned}
$$

The measured value of the $W$-boson mass is compatible with the current world average of $m_{W}=$ $80385 \pm 15 \mathrm{MeV}$. The uncertainty is competitive with the current most precise measurements performed by the CDF and D0 collaborations.

Corfu Summer Institute 2017 'School and Workshops on Elementary Particle Physics and Gravity' 2-28 September 2017

Corfu, Greece 


\section{Introduction}

After the discovery of the $W$ boson at the CERN SPS Collider in 1983, efforts have been directed towards the determination of its properties. In particular, precise knowledge of the $W$-boson mass is of a high importance for testing the consistency of the Standard Model, as it is connected with relatively well-measured top quark and the Higgs boson masses via loop-induced radiative corrections. Increasing the precision of the $W$-boson mass measurement probes new physics, as the predicted $W$-boson mass also receives contributions from additional particles. However, the precise measurement of the $W$-boson mass at hadron colliders remains a major challenge.

The first measurement of the $W$-boson mass was performed during the discovery by the protonantiproton collider at CERN, with a value of $m_{W}=81 \pm 5 \mathrm{GeV}[2,3]$. Later its value was measured with a relative error better than $1 \%$ by the experiments of the electron-positron collider LEP at CERN and of the proton-antiproton collider Tevatron at Fermilab. Measurements performed by the LEP and Tevatron collider experiments have reduced the precision considerably. Now the combined value is $m_{W}=80370 \pm 15 \mathrm{MeV}$ [4] which corresponds to a relative accuracy of $0.02 \%$. At present, the most precise value is obtained with the CDF detector at Fermilab [5]. On the other hand, the value of $m_{W}$ is predicted with a precision of the order of $0.01 \%$ [6], which dictates the desired precision for direct measurements to be able to test the Standard Model.

At the LHC, $W$ bosons are produced mainly via the annihilation of a quark and an antiquark. In contrast to the production of $W$ bosons in proton-antiproton collisions at Tevatron, where valence antiquarks participate, at the LHC only sea antiquarks, derived from gluon separation, can produce $W$ bosons. Knowledge of proton distribution functions (PDFs) for sea quarks, and particularly for the quarks of second generation, is therefore of a particular importance. To achieve a measurement accuracy similar to Tevatron, it is necessary to constrain the corresponding PDF uncertainties using the LHC data.

Another difficulty in measuring $m_{W}$ is related to the large number of simultaneous inelastic interactions occurring at each LHC beam crossing. These additional interactions considerably complicate the reconstruction of the particles in the ATLAS detector [1], and only the leptonic decays of the $\mathrm{W}$ can be reconstructed with sufficient efficiency and reduced background contributions. The leptonic decay products used for the present measurement are an electron or a muon accompanied by a neutrino. In fact, the neutrino escapes detection, but its presence is detected by momentum imbalance of visible particles in an event in the plane transverse to the beams. Its transverse momentum, $p_{\mathrm{T}}^{v}$, can be measured indirectly from the lepton and from the hadron system which recoils the $W$ boson. All the kinematic variables are thus considered in the transverse plane.

\section{Methodology}

There are three kinematic variables which are sensitive to the $W$-boson mass [11]:

- transverse momentum of the charged lepton $p_{\mathrm{T}}^{\ell}$

- missing transverse energy $E_{\mathrm{T}}^{\text {miss }}=p_{\mathrm{T}}^{v}$

- transverse mass of the lepton-neutrino system $m_{\mathrm{T}}^{W}=\sqrt{2 p_{\mathrm{T}}^{\ell} E_{\mathrm{T}}^{\text {miss }} \cdot\left(1-\cos \left(p_{\mathrm{T}}^{\ell}, E_{\mathrm{T}}^{\text {miss }}\right)\right)}$ 

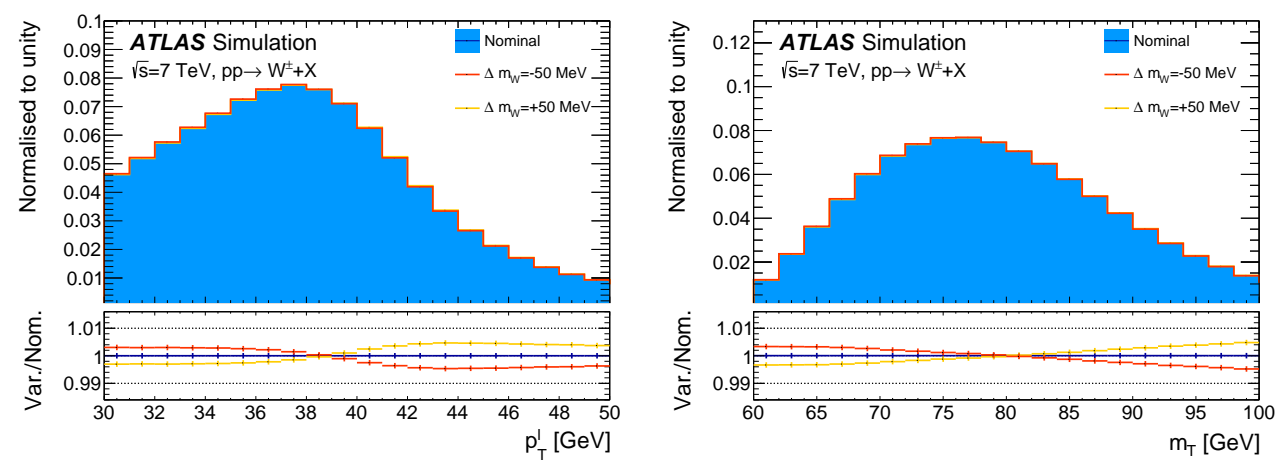

Figure 1: Kinematic distributions of $p_{\mathrm{T}}^{\mu}$ (a) and $m_{\mathrm{T}}^{W}$ (b) in simulated events for the $W$-boson mass fitted value $m_{W}=80370 \mathrm{MeV}$ and the shifted values $m_{W}=80320 \mathrm{MeVand} m_{W}=80420 \mathrm{MeV}$.

The determination of the $W$-boson mass exploits the kinematic peaks of these distributions. In particular, in the rest frame of decaying $W$ boson, the charged lepton and the neutrino are backto-back, so the lepton transverse momentum and missing transverse energy spectra have Jacobian peaks at $p_{\mathrm{T}}=m_{W} / 2$, whilst the transverse mass spectrum peaks at $m_{\mathrm{T}}^{W}=m_{W}$. These peaks are additionally smeared out by non-zero $p_{\mathrm{T}}^{W}$ distribution and by detector effects. Therefore, the accurate modeling of the $p_{\mathrm{T}}^{W}$ and precise understanding of the detector performance is required.

The basic idea of the $m_{W}$ determination is to use the correlation between the $W$-boson mass and the resulting energy and momentum of its decay products. Technically this is realised by a so-called template fit approach. For different assumed $W$-boson masses, the expected final state distributions, i.e. templates, which would be measured in the detector are calculated and compared to the actual measurement. The minimal residual difference between the different simulated $m_{W}$ hypotheses and the measurement then provides a handle on the actual $W$-boson mass. This basic principle is illustrated in Figure 1, where $p_{\mathrm{T}}^{\mu}$ and $m_{\mathrm{T}}^{W}$ templates for three significantly different masses are compared.

This method is sensitive to various sources of uncertainty. Typical examples are the efficiency of lepton reconstruction, the calibration of the transverse momentum of the leptons and of the missing transverse energy, the background noise, and the mechanism of $W$-boson production and decay. Such effects can introduce a bias in the measurement and must be sufficiently controlled.

\section{Experimental corrections}

The ATLAS simulation already includes the knowledge of the detector geometry, material distribution, and physics model of the lepton interactions with detector, which were studied during previous years. However, additional corrections are needed to compensate the remaining mismodelings at the highest possible level of precision. The lepton momentum scale and resolution obtained from the reconstruction of MC simulated events need to be corrected to precisely reproduce the characteristics of the lepton momentum reconstructed in real data. The corresponding correction factors were derived from studying the mass distribution of dilepton resonance $Z \rightarrow \ell^{+} \ell^{-}$ $\left(m_{\ell \ell} \approx 91.2 \mathrm{GeV}\right)$ which is available with high statistics. Electron energy and muon momentum scale corrections are obtained by exploiting the peak position of the invariant mass distribution of 

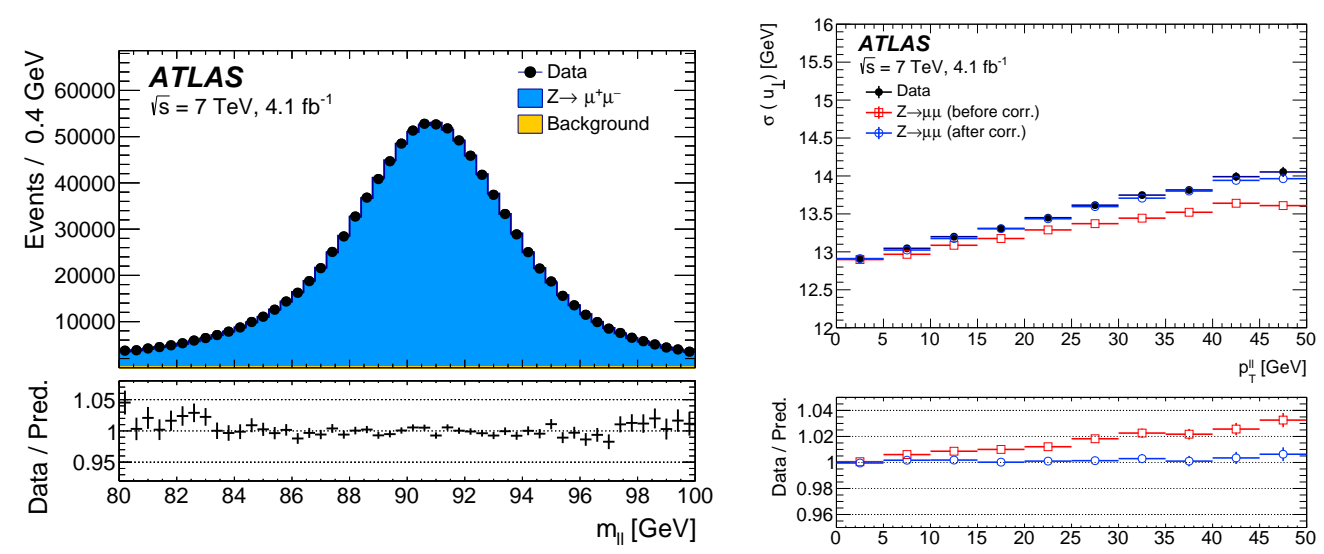

Figure 2: Left: dimuon invariant mass distribution in $Z \rightarrow \ell^{+} \ell^{-}$events. Corrections for momentum scale and resolution, and for reconstruction, isolation, and trigger efficiencies are applied to the muons in the simulated events. Right: RMS of $u_{\perp}$ distributions as a function of $p_{\mathrm{T}}^{\ell \ell}$ in data and simulated $Z \rightarrow \mu^{+} \mu^{-}$ events before and after the hadronic recoil corrections are applied.

the lepton pairs in the final state around the mass $m_{Z}$. In addition, the energy resolution of the electrons and momentum resolution of muons are corrected by requiring an exact description of the data by the simulation as shown in Figure 2 (left).

The efficiency of the detector to trigger and reconstruct leptons, and the efficiency to select isolated leptons (i.e. no significant activity around the lepton tracks), in particular the dependence on the lepton momenta and pileup conditions, has to be also taken into account in the modeling. In case of electrons, the selection of identification is additionally applied in order to reject the background coming from hadrons. This selection uses cuts on nine variables that characterize the lateral and longitudinal development of the electromagnetic shower of electrons in the calorimeter, and certain criteria related to the quality of the associated tracks. This is implemented in additional class of corrections: efficiency corrections. These were obtained with so-called Tag-and-Probe method $[14,15]$. In this analysis the corrections were calculated separately in different categories of lepton pseudorapidity $\eta^{\ell}$ and $p_{\mathrm{T}}^{\ell}$ in order to match data and simulation with sufficient precision.

Another quantity that has to be calibrated is the hadronic recoil in the transverse plane. The hadronic recoil is defined as the vector sum of the transverse energy of all clusters reconstructed in the calorimeters, excluding energy deposits associated with the decay leptons: $\vec{u}_{\mathrm{T}}=\sum \vec{E}_{\mathrm{T}, \mathrm{i}}$. The hadronic recoil is calibrated using an expected balance between recoil $u_{\mathrm{T}}$ and transverse momentum $p_{\mathrm{T}}^{\ell \ell}$ of two decay leptons of $Z$ boson. In particular, the projections of the recoil onto the axes parallel $\left(u_{\|}^{Z}\right)$ and perpendicular $\left(u_{\perp}^{Z}\right)$ to the $Z$-boson transverse momentum are sensitive to the response and resolution of the detector. Figure 2 (right) illustrates the recoil resolution as a function of $p_{\mathrm{T}}^{\ell \ell}$ in data and simulated $Z \rightarrow \mu^{+} \mu^{-}$events before and after the hadronic recoil corrections are applied. The corrections derived from $Z$ boson are then propagated to $W$ bosons.

Experimentally, the $p_{\mathrm{T}}^{\ell}$ distribution is mainly affected by the calibration of charged leptons, while the $E_{\mathrm{T}}^{\text {miss }}$ distribution is sensitive to the calibration of the hadronic recoil $u_{\mathrm{T}}$. The distribution of $m_{\mathrm{T}}^{W}$ is constructed using $p_{\mathrm{T}}^{\ell}$ and $E_{\mathrm{T}}^{\text {miss }}$, and is therefore also affected by both effects. A good agreement between the $m_{W}$ values from the different distributions validates the applied experimen- 
tal corrections.

\section{Theoretical corrections}

The study of the production of $W$ and $Z$ vector bosons is affected by significant complications related to the strong interaction. The transverse momentum of the $W$ and $Z$ bosons is non-zero due to emission of quarks and gluons from the initial-state partons. Majority of the events are at low values of boson transverse momentum where large logarithms need to be resummed and non-perturbative effects must be included to fixed order perturbative QCD calculations either with parton shower models or predictions based on analytical resummation. Simultaneously, higherorder corrections to initial-state QCD interactions affect the vector boson polarization states and introduce asymmetries in the angular distribution of the decay leptons. Thus, the distribution of $p_{\mathrm{T}}^{\ell}$ is broadened by the distribution of the boson $p_{\mathrm{T}}$, and is sensitive to the $W$-boson helicity states, in turn affected by the proton PDFs.

The samples used in this analysis are simulated with the PowHEG+PYTHIA generator. However, to achieve sufficient accuracy of the $m_{W}$ measurement, the missing higher-order QCD and EW effects from specialized tools must be included in the simulation. Physics corrections to the final state distributions predicted in these samples are based on the ansatz of the factorization of the cross section in four pieces, according to the following formula:

$$
\frac{d \sigma}{d p_{1} d p_{2}}=\left(\frac{d \sigma(m)}{d m}\right)\left(\frac{d \sigma(y)}{d y}\right)\left(\frac{d \sigma\left(p_{\mathrm{T}}, y\right)}{d p_{\mathrm{T}}} \frac{1}{\sigma(y)}\right)\left(\Sigma_{i} A_{i}\left(y, p_{\mathrm{T}}\right) P_{i}(\cos \theta, \phi)\right),
$$

where $p_{1}$ and $p_{2}$ are the lepton and anti-lepton 4-momenta, $m, p_{\mathrm{T}}$, and $y$, are the invariant mass, transverse momentum, and rapidity of the dilepton system, $\cos \theta$ and $\phi$ are the polar and azimuthal angle of the lepton in a given rest frame of the dilepton system, $A_{i}$ are angular coefficients, and $P_{i}$ are spherical harmonics.

Variations of $\frac{d \sigma(m)}{d m}$ with respect to the the value of the pole mass of the $W$ boson are modeled with a Breit-Wigner distribution, including EW corrections. The differential cross section $\frac{d \sigma}{d y}$, and the $A_{i}\left(y, p_{\mathrm{T}}\right)$ angular coefficients are modeled with perturbative NNLO QCD predictions evaluated with DYNNLO based on CT10nnlo PDF [7]. The transverse momentum spectrum at given rapidity, $\frac{d \sigma(p t, y)}{d p t} \frac{1}{\sigma(y)}$, is modeled with parton shower using PYTHIA 8. In addition, the samples are corrected using the PHOTOS code [8] to take into account the effects of QED affecting the final state.

Various ancillary measurements of $W$ - and $Z$-boson production are used to validate and constrain the modelling of the fully-differential leptonic Drell-Yan cross section. The PDF central values and uncertainties, as well as the modelling of the $Z$-boson differential cross section as a function of rapidity and the $W$-boson differential cross section as a function of lepton pseudorapidity, are validated by comparing to the corresponding $7 \mathrm{TeV} W$ - and Z-boson cross section measurements, based on the same data sample. The comparison with the measured $\mathrm{W}$ and $\mathrm{Z}$ cross sections is shown in Figure 3 where satisfactory agreement between the measurements and theoretical predictions is observed.

The QCD parameters of the parton shower PYTHIA 8 model are determined by fits to the transverse-momentum distribution of the $Z$ boson measured at $7 \mathrm{TeV}$ [9], resulting into so-called Pythia 8 AZ tune (see Figure 4 (left)). Three QCD parameters were considered in the fit: the 

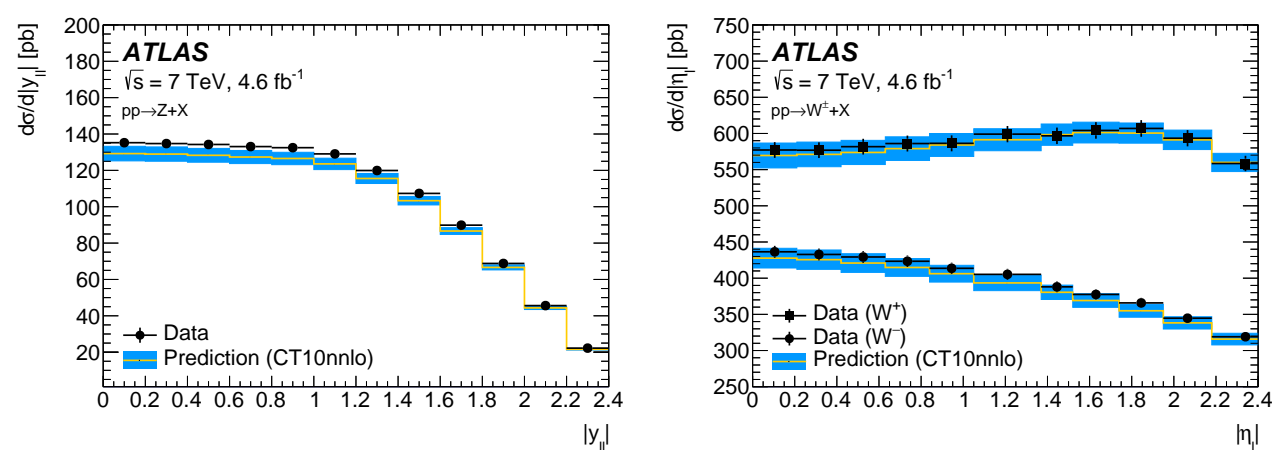

Figure 3: Left: differential Z-boson cross-section as a function of boson rapidity, and (right) differential $W^{+}$and $W^{-}$cross-sections as a function of charged decay-lepton pseudorapidity at $\sqrt{s}=7 \mathrm{TeV}$ [12]. The measured cross sections are compared to the DYNNLO predictions using the CT10nnlo PDF set. The vertical bars show the total experimental uncertainties, including luminosity uncertainty, and the bands show the PDF uncertainties of the predictions.
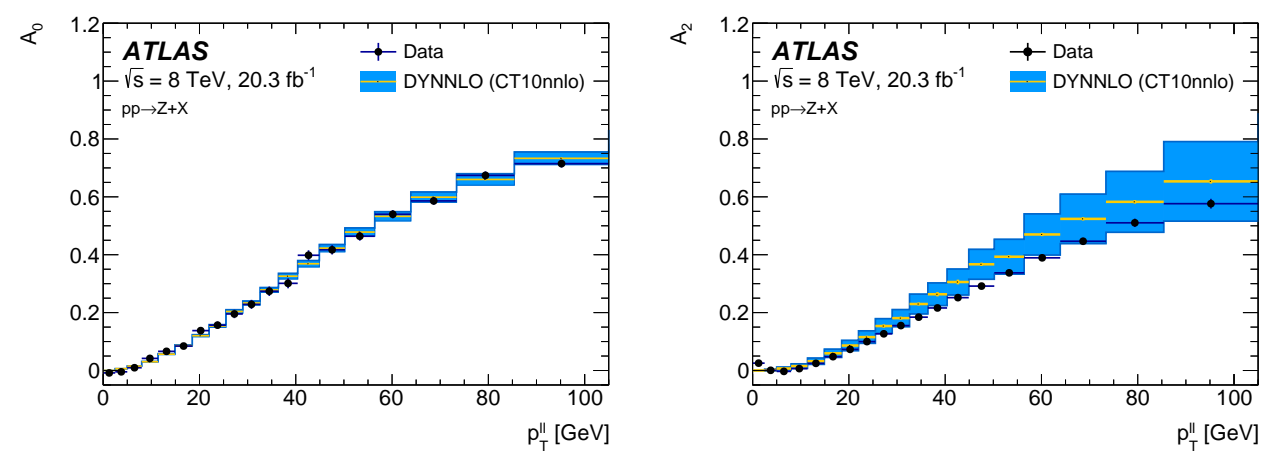

Figure 4: Measured differential cross section of $p_{\mathrm{T}}^{Z}$ (left) and the differential cross-section ratio of $\sigma(W) / \sigma(Z)$ vs. $p_{\mathrm{T}}^{V}(\mathrm{~V}=\mathrm{W}, \mathrm{Z})($ right $)$ compared to the PowHEG+PythIA prediction, reweighted to the $\mathrm{AZ}$ tune. Statistical and experimental systematic uncertainties are indicated.

intrinsic transverse momentum of the incoming partons, the value of the strong coupling constant at the Z-boson mass used for the QCD ISR, and the value of the ISR infrared cut-off. These predictions were used to model the spectrum of $p_{\mathrm{T}}^{W}$. For further validation, the predicted differential cross-section ratio $R_{W / Z}\left(p_{\mathrm{T}}\right)$ is compared with PYTHIA $8 \mathrm{AZ}$ to the corresponding ratio of ATLAS measurements of vector-boson transverse momentum, as shown in Figure 4 (right). Theoretical predictions are found to be in agreement with the experimental measurements.

Modelling of the $A_{i}\left(p_{\mathrm{T}}, y\right)$ coefficients is validated by comparing the theoretical NNLO predictions to the $8 \mathrm{TeV}$ measurement of the angular coefficients in Z-boson decays [13]. Good greement between the measurements and DYNNLO is observed for all coefficients, except for $A_{2}$. Figure 5 exemplifies the comparison for $A_{0}$ and $A_{2}$ as a function of $p_{\mathrm{T}}^{Z}$. The observed disagreement with data for $A_{2}$ is considered as additional source of uncertainty in the theoretical prediction. These predictions are then extrapolated to the production of $W$ bosons.

Uncertainties associated with missing higher order electroweak corrections were estimated using the WINHAC code [10], and found to contribute to about $5.5 \mathrm{MeV}$ when using the $p_{\mathrm{T}}^{\ell}$ ob- 

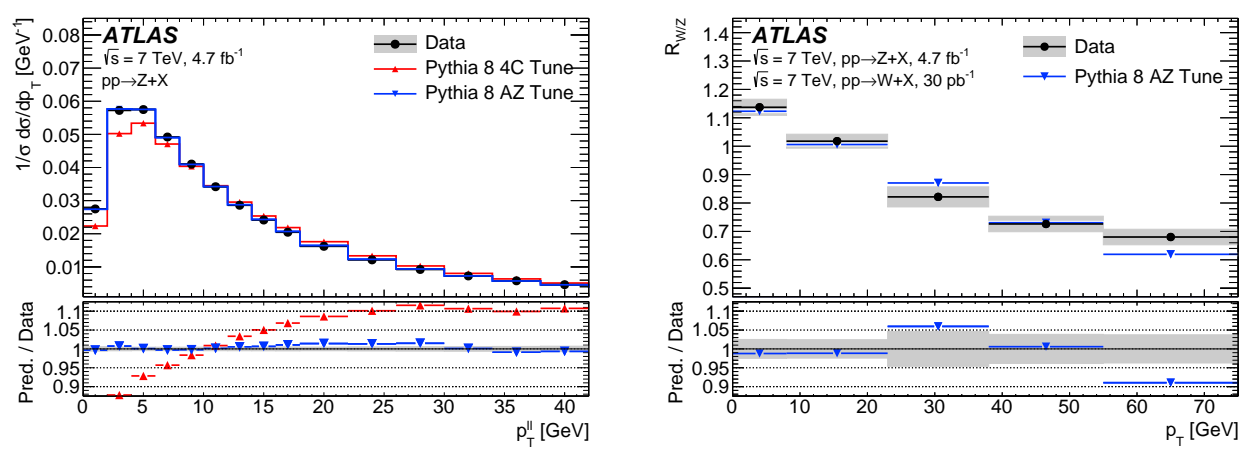

Figure 5: The $A_{0}$ (left) and $A_{2}$ (right) angular coefficients in $Z$-boson events as a function of $p_{\mathrm{T}}$. The measured coefficients are compared to the DYNNLO predictions using the CT10nnlo PDF set. The vertical bars show the total experimental uncertainties, and the bands show the uncertainties assigned to the theoretical predictions.

servable, and $2.5 \mathrm{MeV}$ when using $m_{\mathrm{T}}$. These uncertainties are presently sub-leading. PDF is the dominant source of uncertainty and contributes about 8.0 and $8.7 \mathrm{MeV}$ for charge-combined lepton transverse momentum and transverse mass fits, respectively, when using the nominal CT10 PDF for the estimation (with uncertainty variations scaled down to cover $68 \% \mathrm{CL}$ ). Envelope from comparison of CT10nnlo to CT14 and MMHT2014 PDF sets additionally contributes to about $3.8 \mathrm{MeV}$. The effect of missing higher-order corrections on the NNLO predictions was estimated by varying the renormalisation and factorisation scales by factors of 0.5 and 2.0 and found to be small. Several sources of uncertainty affecting the $p_{\mathrm{T}}^{W}$ distribution are the accuracy of $p_{\mathrm{T}}^{Z}$ fit (about $3 \mathrm{MeV}$ ), variation of charm-quark mass (about $1 \mathrm{MeV}$ ), uncertainty due to missing higher-order QCD effects estimated through variations of the factorisation scale $\left(5.0 \mathrm{MeV}\right.$ for $p_{\mathrm{T}}^{\ell}$ and $6.9 \mathrm{MeV}$ for $\left.m_{\mathrm{T}}\right)$, parton shower PDF uncertainty (about $1 \mathrm{MeV}$ ). Finally, polarization uncertainties are about $6.4 \mathrm{MeV}$, with 6.2 MeV contributed by the $Z$ data accuracy, and $1.6 \mathrm{MeV}$ from the discrepancy observed for $A_{2}$.

\section{Background modeling}

The $W \rightarrow \ell v$ candidates with leptonic final states are contaminated with relatively low background. The expected background results from $W \rightarrow \tau v, Z \rightarrow \ell \ell, Z \rightarrow \tau \tau$, as well as dibosons decays, top processes and multijet events. The electroweak and top processes $(t \bar{t}$, as well as single $t$ production) are modeled with good accuracy: their cross-sections are measured with good agreement with the theoretical expectations, and Monte Carlo is used to implement them.

In contrast, the multijet background cannot be simulated reliably. It is therefore necessary to use a data-driven method for the multijet background estimate. In this method, the multijet background sample is constructed from a jet-enriched control region in data by reversing or relaxing some of the lepton isolation requirements. Such control region is assumed to provide a shape of the background for all variables used for the $m_{W}$ extraction. The normalisation for the multijet sample is determined from the fraction fit method using several discriminating variable $\left(E_{\mathrm{T}}^{\mathrm{miss}}\right.$, $\left.m_{\mathrm{T}}^{W}, p_{\mathrm{T}}^{\ell} / m_{\mathrm{T}}^{W}\right)$ in a region where kinematic cuts are removed. The relative normalizations of events containing a true lepton from the $W$ signal or the multijet background noise are adjusted to reproduce the distributions observed in data as shown in Figure 6 (left). The contribution from simulated 

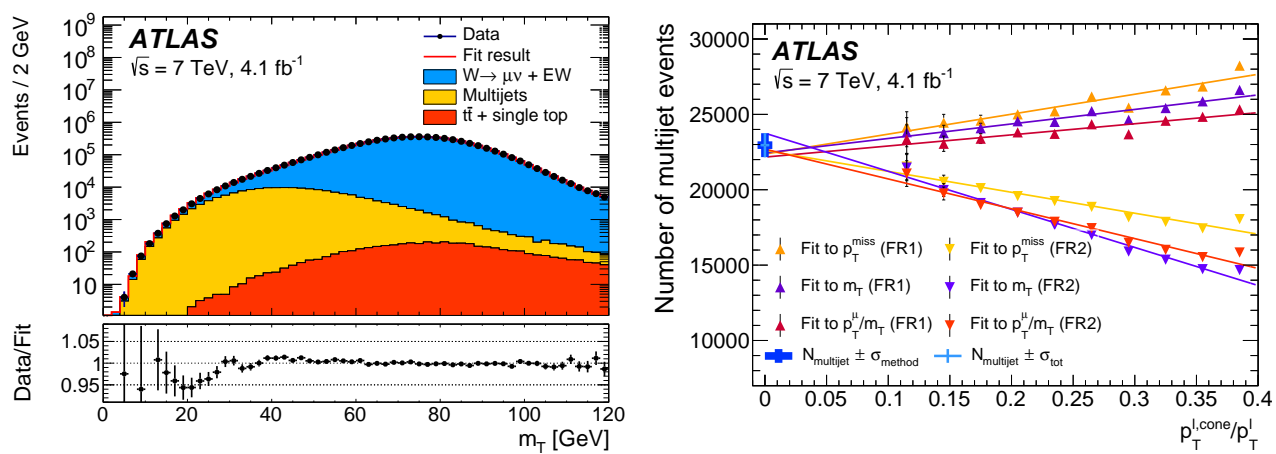

Figure 6: Left: example of multijet background fit to the $E_{\mathrm{T}}^{\text {miss }}$ distribution in the region with relaxed $E_{\mathrm{T}}^{\text {miss }}>30 \mathrm{GeV}$ and $m_{\mathrm{T}}^{W}>60 \mathrm{GeV}$ cuts. Multijet distribution is extracted from data by requiring $0.2<$ $p_{\mathrm{T}}^{\mu, \text { cone }} / p_{\mathrm{T}}^{\mu}<0.4$, where $p_{\mathrm{T}}^{\mu \text {,cone }}$ is the scalar sum of the $p_{\mathrm{T}}$ of tracks in a cone of size $\Delta R<0.2$. Right: estimated number of multijet-background events as a function of the lower bound of the isolation variable range used to define the control regions.

background events is accounted using the simulation. The correlation between the kinematic variables and the lepton isolation is taken into account by splitting the samples using different lepton isolation criteria. Afterwards, the procedure of background estimates is repeated in each isolation sub-sample using three kinematic variables and two fitting regions (see Figure 6 (right)). Finally, the results are extrapolated to the isolated region and the multijet background yield is determined from spread of the linear extrapolations. The impact of the multijet background systematic uncertainties on the determination of $m_{W}$ is about $4 \mathrm{MeV}$ in muon channel and $8 \mathrm{MeV}$ in electron channel. The largest source of uncertainty on simulated background is due to modeling of $Z \rightarrow \ell \ell$ background and corresponds to about $4 \mathrm{MeV}$.

\section{Results}

The measured value of $m_{W}$ is obtained from the combination of different measurements performed in the electron and muon decay channels, separately for positively and negatively charged $W$ bosons, and in different lepton pseudorapidity categories. The $m_{W}$ measurements in each category are based on the $p_{\mathrm{T}}^{\ell}$ and $m_{\mathrm{T}}^{W}$ distributions, which are partially correlated. In total there are 28 measurement categories. Figure 7 and Figure 8 show the $p_{\mathrm{T}}^{\ell}($ left $)$ and $m_{\mathrm{T}}^{W}$ (right) distributions for $W^{-}$events in electron and muon channels respectively, after all experimental and theoretical corrections are applied. The compatibility of the results in the electron and muon channels provides a test of the experimental corrections, while the compatibility of the results measured in the pseudorapidity categories $\left|\eta_{\ell}\right|$ and separately for $W^{+}$and $W^{-}$tests the theoretical modeling. Further tests are performed by measuring $m_{W}$ in categories of the average number of simultaneous inelastic interactions, in two intervals of hadronic recoil, as well as without applying a cut on $E_{\mathrm{T}}^{\text {miss }}$. These tests allow to check the calibration of the hadronic recoil and the modeling of the transverse momentum distribution of the $W$ boson.

In addition, theoretical and experimental corrections were checked with the $Z \rightarrow \ell \ell$ events by measuring $m_{Z}$ using the same method. The determination of $m_{Z}$ from the invariant mass of the lepton pairs provides a first test of the energy calibration of the leptons. Moreover, the determination 



Figure 7: The $p_{\mathrm{T}}^{\ell}$ (left) and $m_{\mathrm{T}}^{W}$ (right) distributions for $W^{-}$events in the electron decay channel. The data are compared to the simulation including signal and background contributions. Detector calibration and physics-modelling corrections are applied to the simulated events. The value of $m_{W}$ is set according to the overall measurement result. The ratio panels show the data-to-prediction ratios, the error bars show the statistical uncertainty, and the band shows the systematic uncertainty of the prediction.


Figure 8: The $p_{\mathrm{T}}^{\ell}$ (left) and $m_{\mathrm{T}}^{W}$ (right) distributions for $W^{-}$events in the muon decay channel. The data are compared to the simulation including signal and background contributions. Detector calibration and physics-modelling corrections are applied to the simulated events. The value of $m_{W}$ is set according to the overall measurement result. The ratio panels show the data-to-prediction ratios, the error bars show the statistical uncertainty, and the band shows the systematic uncertainty of the prediction.

of $m_{Z}$ from $p_{\mathrm{T}}^{\ell}$ distribution tests the efficiency corrections, the modeling of the transverse momentum of $Z$ boson and its polarization. Finally, $m_{Z}$ measurements using $m_{\mathrm{T}}^{Z}$ and $E_{\mathrm{T}}^{\mathrm{miss}}$ observables, which are defined by treating one of the two decay leptons as a neutrino, provide a test of the recoil calibration.

All the individual $m_{W}$ measurements are found to be consistent and their combination leads to a value of

$$
\begin{aligned}
m_{W} & =80370 \pm 7 \mathrm{MeV} \text { (stat. }) \pm 11 \mathrm{MeV} \text { (exp.sys.) } \pm 14 \mathrm{MeV} \text { (mod.sys.) } \\
& =80370 \pm 19 \mathrm{MeV} .
\end{aligned}
$$

The combined value of the $W$-boson mass is compatible with the current world average of $m_{W}=$ $80385 \pm 15 \mathrm{MeV}$ and with earlier results as illustrated in Figure 10. The uncertainty is competitive to the current most precise measurements performed by the CDF and D0 collaborations. The 

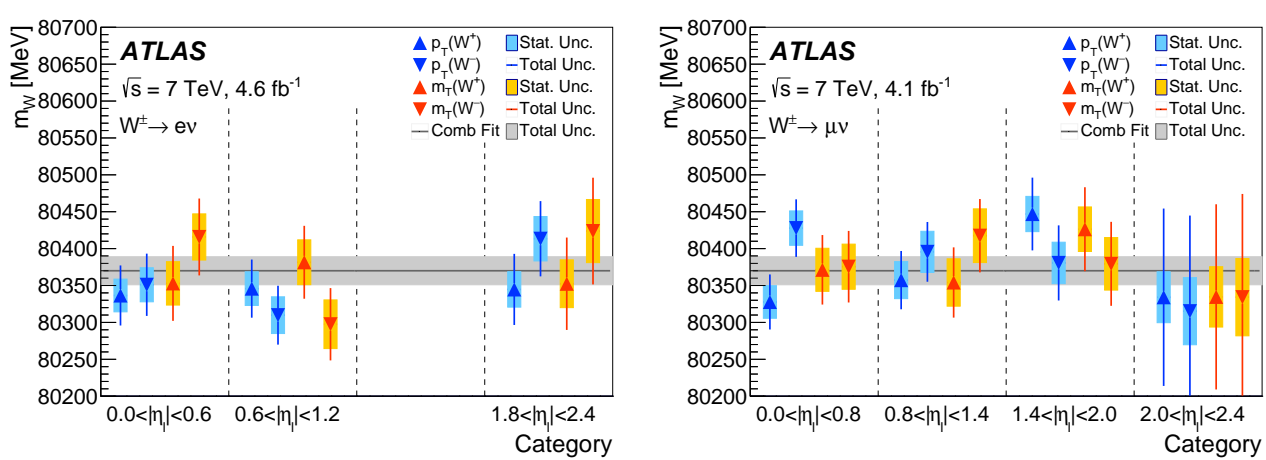

Figure 9: Results of individual $m_{W}$ measurements in the electron (left) and muon (right) decay channels. The coloured bands and solid lines show the statistical and total uncertainties, respectively. The horizontal line and band show the fully combined result and its uncertainty.

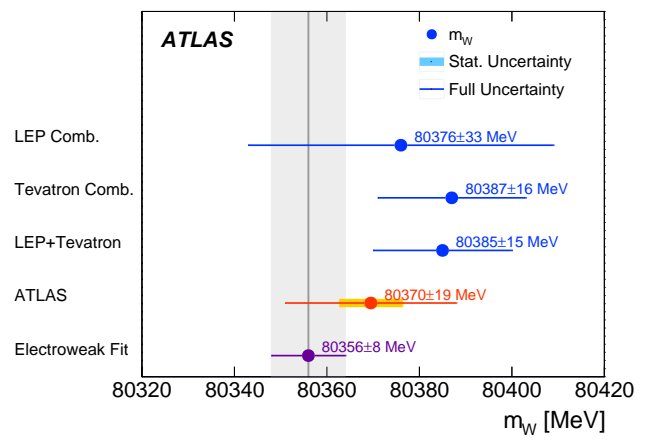

Figure 10: The measured values of $m_{W}$ (red dots) are compared to the SM prediction from the electroweak fit (violet dot), and to the combined values of $m_{W}$ measured at LEP and at the Tevatron collider (blue dots). The light grey band shows the uncertainty of the SM prediction from the electroweak fit, assuming $m_{\mathrm{T}}=173.34 \pm 0.76 \mathrm{GeV}$ and $m_{H}=125.09 \pm$ $0.24 \mathrm{GeV}$.

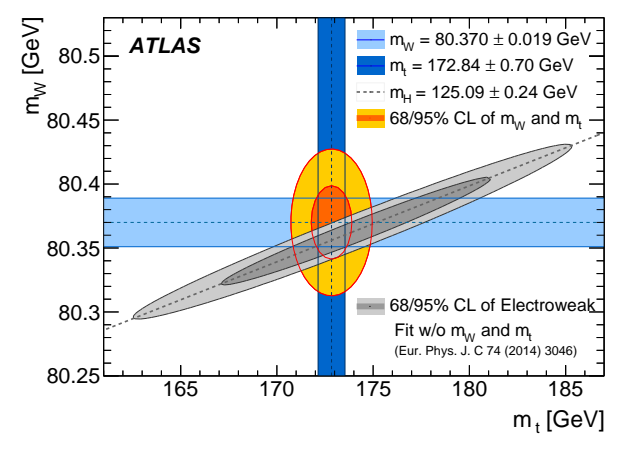

Figure 11: The $68 \%$ and $95 \%$ confidence level contours of the $m_{W}$ and $m_{\mathrm{T}}$ indirect determination from the global electroweak fit (grey ellipses) are compared to the $68 \%$ and $95 \%$ confidence level contours of the ATLAS measurements of the top-quark and $W$-boson masses (red and orange ellipses). The determination from the electroweak fit uses as input the LHC measurement of the Higgs boson mass, $m_{H}=$ $125.09 \pm 0.24 \mathrm{GeV}$. The light and dark blue bands indicate the uncertainties on the ATLAS measurements of $m_{W}$ and $m_{\mathrm{T}}$.

measured value is consistent with the SM expectation of $m_{W}=80358 \pm 8 \mathrm{MeV}$ obtained from the electroweak fit using recent measurements of the top-quark and Higgs boson masses as illustrated in Figure 11.

\section{References}

[1] ATLAS Collaboration, The ATLAS Experiment at the CERN Large Hadron Collider, JINST 3 (2008) S08003. 
[2] UA1 Collaboration, G.Arnison et al., Experimental observation of isolated large transverse energy electrons with associated missing energy at $\sqrt{s}=540 \mathrm{GeV}$, Phys. Lett. B 122 (1983) 103-116.

[3] UA2 Collaboration, M.Banner et al., Measurement of the $W$-boson mass in pp collisions at $\sqrt{s}=7$ TeV with the ATLAS detector, Phys. Lett. B 122 (1983) 476-485.

[4] Particle Data Group, K.A.Olive et al., Chin. Phys. C 38 (2014) 090001.

[5] CDF, Aaltonen, T. and others, Precise measurement of the W-boson mass with the CDF II detector, Phys. Rev. Lett. 108 (2012) 151803 [arXiv: 1203 . 0275].

[6] Gfitter Group, M.Baak et al., The global electroweak fit at NNLO and prospects for the LHC and ILC, Eur. Phys. J. 108 (2014) 3046 [arXiv: 1407 . 3792].

[7] S. Catani et al., Vector boson production at hadron colliders: a fully exclusive QCD calculation at NNLO, Phys. Rev. Lett. 103 (2009) 082001 [arXiv: 0903.2120 ].

[8] Golonka, Piotr and Was, Zbigniew, PHOTOS Monte Carlo: A Precision tool for QED corrections in Z and $W$ decays, Eur. Phys. J. C45 (2006) 97-107 [arXiv: hep-ph/ 0506026 ].

[9] ATLAS Collaboration, Aad, Georges et al., Measurement of the $Z / \gamma^{*}$ boson transverse momentum distribution in pp collisions at $\sqrt{s}=7$ TeV with the ATLAS detector, JHEP 09 (2014) 145 [arXiv:1406.3660].

[10] Płaczek, W. and Jadach, S. and Krasny, M. W., Drell-Yan processes with WINHAC, Acta Phys. Polon. B44 (2013) 2171-2178 [arXiv: 1310 .5994].

[11] ATLAS Collaboration, Measurement of the W-boson mass in pp collisions at $\sqrt{s}=7$ TeV with the ATLAS detector, Eur. Phys. J. C 78 (2018) 110 [arXiv: 1701.07240 ].

[12] ATLAS Collaboration, Precision measurement and interpretation of inclusive $W^{+}, W^{-}$and $Z / \gamma^{*}$ production cross sections with the ATLAS detector, Eur. Phys. J. C 77 (2017) 367 [arXiv: 1612.03016$]$.

[13] ATLAS Collaboration, Measurement of the angular coefficients in Z-boson events using electron and muon pairs from data taken at $\sqrt{s}=8 \mathrm{TeV}$ with the ATLAS detector, JHEP 1608 (2016) 159 [arXiv:1606.00689].

[14] ATLAS Collaboration, Electron reconstruction and identification efficiency measurements with the ATLAS detector using the 2011 LHC proton-proton collision data, Eur. Phys. J. C 74 (2014) 2941 [arXiv: 1404.2240$]$.

[15] ATLAS Collaboration, Measurement of the muon reconstruction performance of the ATLAS detector using 2011 and 2012 LHC proton-proton collision data, Eur. Phys. J. C 74 (2014) 3130 [arXiv: 1407.3935$]$. 